\title{
A Systematic Review on Machine Learning in Neurosurgery: The Future of Decision-Making in Patient Care
}

\author{
Emrah CELTIKCI \\ University of Pittsburgh Medical Center, Department of Neurological Surgery, Pittsburgh, PA, USA
}

\section{ABSTRACT}

Current practice of neurosurgery depends on clinical practice guidelines and evidence-based research publications that derive results using statistical methods. However, statistical analysis methods have some limitations such as the inability to analyze nonlinear variables, requiring setting a level of significance, being impractical for analyzing large amounts of data and the possibility of human bias. Machine learning is an emerging method for analyzing massive amounts of complex data which relies on algorithms that allow computers to learn and make accurate predictions. During the past decade, machine learning has been increasingly implemented in medical research as well as neurosurgical publications. This systematical review aimed to assemble the current neurosurgical literature that machine learning has been utilized, and to inform neurosurgeons on this novel method of data analysis.

KEYWORDS: Bayesian network, Logistic regression analysis, Machine learning, Neural network, Neurosurgery, Support vector machine

\section{INTRODUCTION}

$\mathrm{C}$ urrent decision-making process in neurosurgery is based on clinical practice guidelines that cite wide sample clinical trials and case series publications. Common statistical methods are utilized in the majority of these publications such as; t-test, cox-hazard model and chisquare test which are also well-known to the neurosurgeons. Statistical tests calculate the correlation between variables and require assumptions as in determining level of significance, e.g. setting a $p$-value. Also, the presence of a correlation between variables does not always prove a causation (2). Most of the statistical tests have some kind limitations such as being unable to analyze non-linear variables or difficulty in finding correlations when data sets are massive.

In the past decades, machine learning $(\mathrm{ML})$ has been proposed as a novel computational data analysis method in order to overcome the limitations of traditional scientific statistical methods and has become increasingly popular amongst medical sciences. ML is a subfield of computer science, and focuses on creating algorithms that allow computers to learn from data. Its ability to perform comprehensive analyses even with massive amounts of non-linear data makes it favorable in medical decision-making. There are different types of learning and different mathematical algorithms applied in ML.

Principally, there are two types of learning; supervised and unsupervised learning. In supervised learning, the aim is to predict the output. In order to achieve the capability of prediction, $M L$ requires a training period, when all inputs and their readily available outputs must be registered. Following the training, the algorithm should be tested to observe the quality of predictions. In this step, the researcher inspects if the predictions of the computer are as accurate as known outputs. If the algorithm performs with a good percentage of correct predictions, it can be employed for making predictions where outputs are not known. For example; in order to build an $\mathrm{ML}$ algorithm which will perform predictions on lumbar spine surgery, pre-surgical clinical data and post-surgical outcome data of patients must be registered as the first step. Then, the researcher must test the algorithm if predictions are in line 
with real patient outcomes. Provided that the predictions are satisfactory, the algorithm can be utilized to predict unknown outcomes. In unsupervised learning, the aim is to predict certain patterns in the data rather than an outcome. Pattern analysis is implemented especially for defining pathophysiological mechanisms of diseases. For example; defining the relation patterns between variables such as imaging characteristics, molecular markers, performance score and amount of cerebral edema in glioma patients requires an unsupervised learning process where predicting an outcome is not the ultimate goal.

As mentioned earlier, different mathematical algorithms have been defined for ML. In logistic regression, inputs and outputs come additively and linearly. However, once established, this method does not allow further addition of alternative variables into the analysis. Decision tree allows performing predictions from different variables even if they are not occurring together. For example, a patient with a motor deficit can have a cerebral mass or subdural hematoma or spinal burst fracture or a herniated disc or a non-neurosurgical condition. Decision tree analysis reveals any possible correlation between these clinical entities. Neural networks have the flexibility of changing input features and adding new types of inputs during data collection. Bayesian networks can predict conditional dependencies of random variables. When symptoms are provided, this algorithm can calculate the probability of various diseases in a subject. Linear discriminant analysis aims to find a linear combination of variables that characterizes or classifies two or more types of objects or events. For example, by using inputs as magnetic resonance imaging (MRI) scans, this algorithm can perform automatized diagnosis of brain tumors. Support vector machines are supervised models that require a set of training data, and have the flexibility of using inputs either the data is linear or non-linear. Support vector machines recently demonstrated the capability of analyzing massive amount of diverse data, e.g., predicting prognosis of stroke patients by analyzing diffusion MRI scans (14).

Recently, ML related studies are progressively increasing in the literature due to its advanced data analyzing capabilities. The aim of this systematical review is to compile the current neurosurgical literature that utilized different $M L$ techniques, and to provide neurosurgeons with an up-to-date overview of this emerging data analysis method.

\section{MATERIAL and METHODS}

'Guidelines of Preferred Reporting Items for Systematic Reviews and Meta-analyses: the PRISMA Statement' was followed while performing this systematic review (37). MEDLINE, and the Cochrane Database of Systematic Reviews was queried using combinations of the following keywords: neurosurgery, machine learning, glioma, spine, skull base, prediction, support vector machine, Bayesian network, decision tree, data mining, neural network. Articles in English language, published from 1 January 1900 to 30 January 2017 were evaluated.

Studies were screened by title and abstract in order to identify relevant articles. Inclusion criteria were; studies that utilized
$M L$ 1) in preoperative planning, 2) in predicting outcomes of either interventions or diseases. Flowchart of article selection is illustrated in Figure 1. Studies 1) that are not related to ML algorithms or neurological sciences, 2) concerning psychiatry, radiology, neurology or specialties other than neurosurgery, 3) on human computer interface, 4) written in a language other than English, 5) with no available full-text were excluded. Also, animal studies, reviews, conference papers, poster presentations, editorials, letters to editor were not included. Full-texts of all included articles were retrieved for further analysis. References of all included full-text articles were reviewed to find any related manuscripts.

\section{RESULTS}

\section{Literature Search}

Following elimination of duplicates, a total of 9098 citations were identified. Following exclusion, 37 full-text articles were examined, and consequently 7 articles were excluded due to unrelated study aim. Five of the excluded studies were focused on automatized tumor detection or anatomical structure segmentation, whereas 2 studies were about automatized prediction of pathological or genetic characteristics without considering outcome or survival. After checking references and related articles of full-text articles, 21 additional articles were found eligible and included into systematic review. Included studies were classified according to topics; hydrocephalus, deep brain stimulation, neurovascular, epilepsy, glioma, radiosurgery, spine, traumatic brain injury. Remaining topics were summarized under 'other'. Distributions of studies by topics were summarized in Table I.

\section{Algorithms}

Amongst 51 studies, following algorithms were identified; neural network $(n=17)$, Bayesian network $(n=11)$, support vector machine $(n=16)$, decision tree $(n=5)$, logistic regression $(n=12)$ and discriminant analysis $(n=2)$. Six studies had employed more than one $\mathrm{ML}$ algorithm. Most common algorithms were neural network (27\%) and support vector machine (25\%).

\section{Hydrocephalus}

There were 2 studies on hydrocephalus. Azimi and Mohammadi used neural network analysis for evaluating endoscopic third ventriculostomy (ETV) success in childhood hydrocephalus (6). Habibi et al. also used neural network analysis to predict the risk of ventriculoperitoneal shunt infection in children with hydrocephalus (23).

\section{Deep Brain Stimulation}

There were 6 studies that utilized ML algorithms in deep brain stimulation studies. The majority of these studies focused on automatized localization of the subthalamic nucleus (STN). Two studies used Bayesian network algorithms for STN targeting $(38,52)$. In the study of Rajpurohit et al., logistic regression was employed to define STN (45), and Wong et al. applied an unsupervised ML technique in order to localize STN (53). Baumgarten et al. used neural network analysis to predict any pyramidal tract side effects during deep brain stimulation (8). 
In an investigation by Muniz et al., three different techniques of ML; neural network analysis, support vector machine and logistic regression analysis were employed in order to evaluate the effect of STN stimulation on ground reaction force during gait (39).

\section{Neurovascular}

There were 6 studies that applied ML techniques in analysis of neurovascular pathologies. The study of Asadi et al. investigated the outcome of arteriovenous malformations (AVMs) following endovascular treatment (4). In the separate studies of Dumont (16) and Dumont et al. (17), the authors tried the same algorithm, which is a neural network model, on different patient populations to predict cerebral vasospasm. In the study of Lo et al. authors combined two ML methods; neural networks with Bayesian network algorithms, to predict clinical outcome of patients following aneurysmal subarachnoid hemorrhage (SAH) (31). One other study employed decision tree analysis to reveal factors that increase the risk of developing aggressive behavior in dural arteriovenous fistulas. They reported the presence of cortical venous drainage as the main risk factor (48).

\section{Epilepsy}

In the literature, there were numerous studies that utilized ML methods in the field of epilepsy. Also, there were related studies that applied $\mathrm{ML}$ techniques to analyze data achieved from electroencephalography recordings, intraoperative surface electrode recordings or intracortical electrode recordings, in order to define neural activity signal patterns of language, seizure and face recognition. Six publications were identified as neurosurgical. Three of them were epilepsy surgery

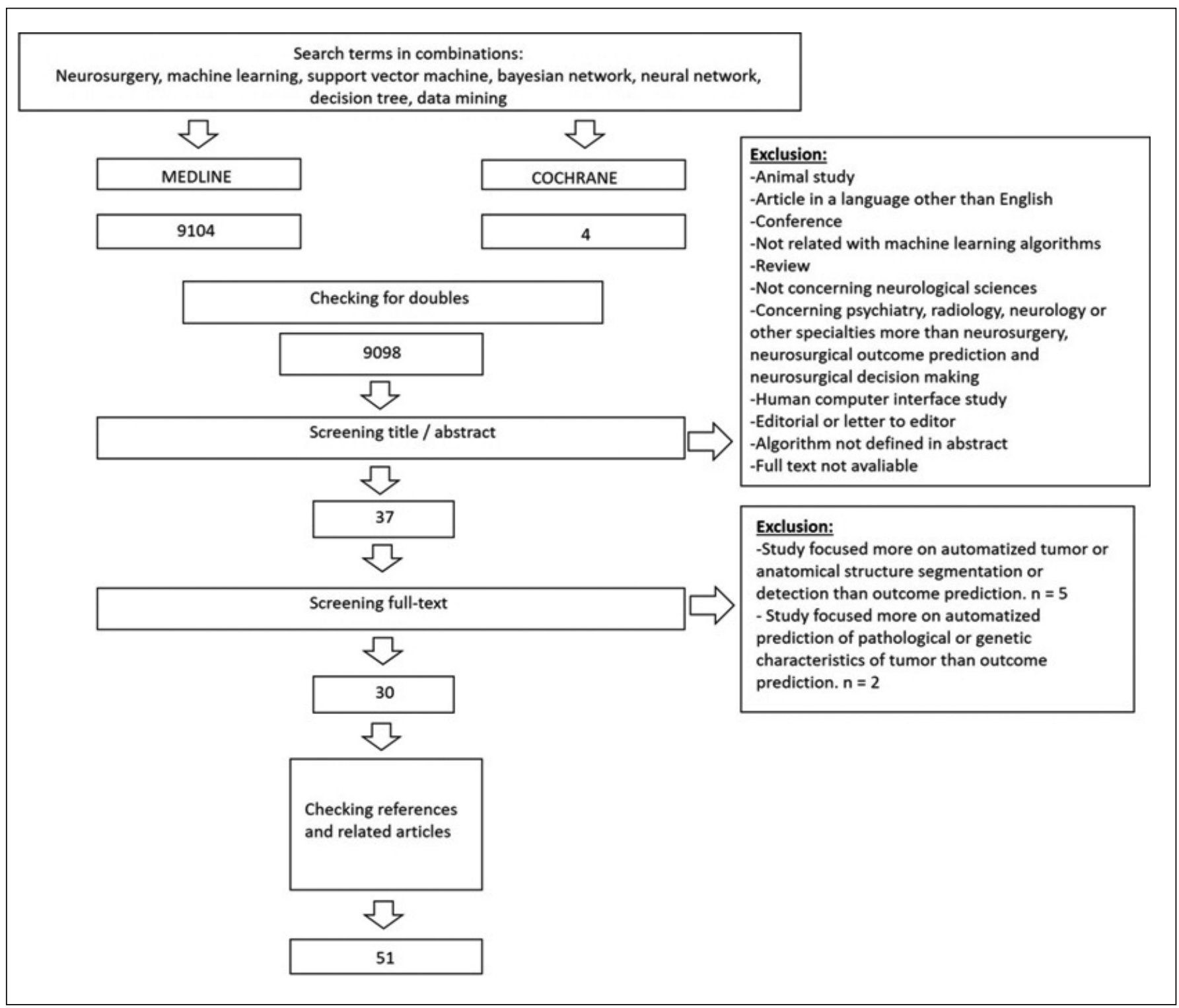

Figure 1: Flow-chart showing selection of relevant articles. 
Table I: Distribution of Studies Included in Systematic Review According to Topics

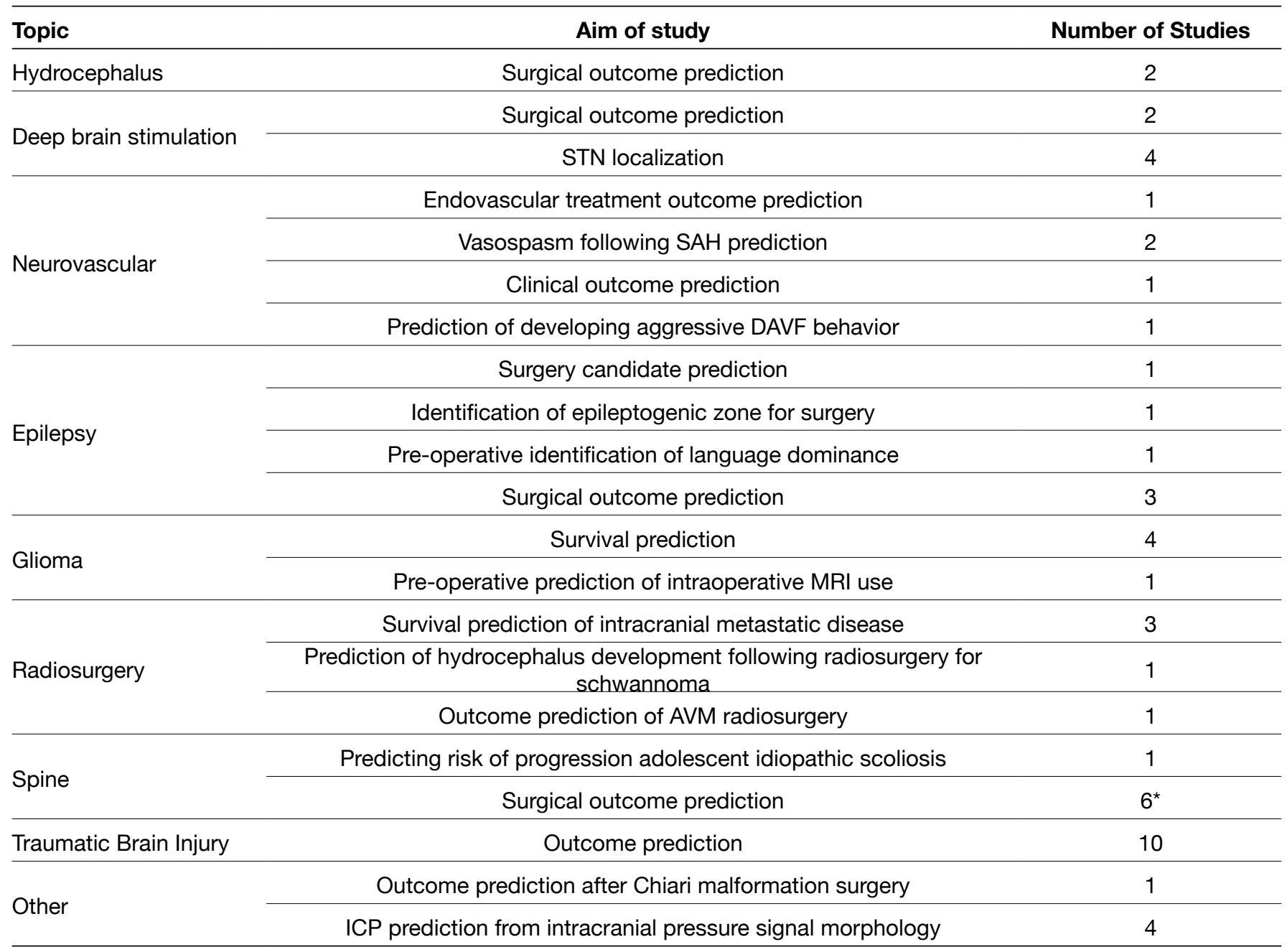

* Two studies out of 6 were about cervical spine, whereas 4 studies were on lumbar spine.

AVM: Arteriovenous malformation, DAVF: Dural arteriovenous fistula, ICP: Intracranial pressure, MRI: Magnetic resonance imaging, SAH: Subarachnoid hemorrhage, STN: Subthalamic nucleus.

outcome prediction studies $(3,9,36)$. Cohen et al. tested if $\mathrm{ML}$ method can identify surgery candidates among intractable temporal lobe epilepsy patients as well as physicians (11). The remaining studies investigated pre-operative identification of the epileptic focus (15), and language dominance (21).

\section{Glioma}

Five studies implemented ML techniques, and 4 of them investigated factors affecting survival in glioma patients $(19,32,33,56)$. One study employed an ML algorithm in order to identify in which patients intraoperative MRI should be used (50). The ML algorithm was given a combined data of surgeon's preoperative opinions and patient's clinical characteristics to make the decision.

\section{Radiosurgery}

Five studies were identified with 3 of them using ML algorithms to predict survival in patients that underwent stereotactic radiosurgery for brain metastases $(10,29,43)$. Oermann et al. used $\mathrm{ML}$ to predict patient outcomes of AVM radiosurgery (43). Another study implemented ML methods to predict the risk of hydrocephalus following gamma knife radiosurgery for intracranial schwannoma (30).

\section{Spine}

There were 7 studies identified, with 6 of them investigated prediction of spinal surgical outcomes via $\mathrm{ML}(5,7,22,24,34,35)$. One study applied support vector machine to predict the risk of progression in adolescent idiopathic scoliosis patients (1).

\section{Traumatic Brain Injury}

Publications on traumatic brain injury that used ML techniques had the greatest number of publications $(n=11)$ with wider sample sizes compared to other topics in this review. These studies employed various ML algorithms such as neural networks, logistic regression model, support vector machine 
and linear discriminant function. All studies, without exception, were designed to investigate outcome prediction $(18,20,25$, $40,41,44,47,51,55,57,58)$.

\section{Other}

Other examples of publications that implemented ML methods for neurosurgical practice were; predicting intracranial pressure (ICP) increase in intensive care unit patients from ICP monitor waveform morphology $(26,27,42,49)$ and predicting the resolution of syringomyelia following Chiari malformation type 1 decompression (13).

\section{- DISCUSSION}

To the best of our knowledge, the current review is the most comprehensive systematic review on neurosurgical literature that employed various ML methods for data analysis. The author believes that this systematic review would provide upto-date information on neurosurgical publications that utilized $M L$ methods while informing neurosurgeons on the subject briefly.

In the past decade, ML was proposed as an alternative method of data analysis, in order to overcome the limitations of linear statistical methods while analyzing and interpreting massive amounts of data. Although ML is not artificial intelligence (Al), it is derived from Al studies which automate analytical building. ML gives computers the ability to learn patterns even when they are non-linear (3). In other fields, ML is employed for tasks like optical character recognition, spam filtering, face recognition, and in search engines. The use of $\mathrm{ML}$ in biological sciences has increased over time due to the limitations of classical statistical methods. Also, statistical methods may cause bias in scientific studies because they require excessive human intervention like choosing the statistical test or assuming the level of significance.

Researchers tend to limit the variables in a study in order to simplify statistical analysis. In contrast, ML techniques can provide high-value predictions without human intervention that may guide better decisions in real time. Furthermore, $\mathrm{ML}$ techniques provide convenience for setting many clinical features, as inputs, and have the ability to analyze the data as a whole. Hoffman et al. compared multivariate linear regression and support vector machine in predicting functional outcome after surgery for cervical spondylotic myelopathy and demonstrated that the ML method was superior to traditional statistical analysis (24). The algorithm used in the study of Oermann et al. was able to provide best predictions of AVM radiosurgery to date (43). Another study by Cohen et al. demonstrated that $M L$ could select epilepsy surgery candidates as good as physicians, with faster evaluation and decision-making (11).

Despite the number of neurosurgical studies using $M L$ are still limited, the increasing trend in this field promises more research publications implementing this technique into decision-making process. This means that some of the clinical guidelines and indications could change in the near future. The study of Knoll et al. provides a clue as to how ML methods could modify our decision-making process (29). The authors retrospectively analyzed 507 patients with intracranial metastases that were treated with radiosurgery in order to determine predictive factors of overall survival. Previously, multiple studies had suggested that the number of metastases $(>4)$ is predictive for overall survival $(1,9,24,25,35)$. However, the $\mathrm{ML}$ technique they employed revealed that performance status and systemic disease status of patients were predictive for overall survival, not the number of intracranial metastases (29). Asadi et al. retrospectively analyzed records of 199 AVM patients and utilized a supervised ML method (LevenbergMarquardt algorithm). They reported that $\mathrm{ML}$ predicted patient outcome with an accuracy of $97.5 \%$ and identified the presence or absence of nidal fistulae as the most important factor (4).

This systematic review only included studies on prediction of patient outcome and survival or pre-operative planning. Also, there are other neurosurgery related studies that implemented $\mathrm{ML}$ and were not included in this review. Radiogenomics studies investigate automatized MRI-based diagnosis of gliomas by establishing the correlation between genetic properties and imaging characteristics of the tumor $(28,46,54)$. Human-computer interface studies like the publication of Collinger et al. is another example, which demonstrated the possibility of a neuroprosthetic arm controlled by intracortical microelectrode implants in the motor cortex (12). In their study, $\mathrm{ML}$ algorithms were not used for predicting any outcome, but employed in order to analyze the complex data acquired from motor cortex recordings.

\section{- CONCLUSION}

$\mathrm{ML}$ provides accurate and fast interpretation of complex data in large amounts, overcoming possible human error and/or bias. This progressively developing method has the ability to learn and gain experience, and would continue to increase its success in accurate decision-making in the future. Wider application of $\mathrm{ML}$ in the medical and neurosurgical studies might improve clinical and surgical management, ultimately the quality of patient care and outcome.

\section{ACKNOWLEDGEMENTS}

I gratefully acknowledge the contributions of Pinar Celtikci, M.D. in critical reviewing, proof-reading and editing of the manuscript. I also thank David T. Fernandes-Cabral M.D. for editing the manuscript and Vijayakrishna Rowthu Ph.D. for providing general advice on the field.

\section{- REFERENCES}

1. Ajemba PO, Ramirez L, Durdle NG, Hill DL, Raso VJ: A support vectors classifier approach to predicting the risk of progression of adolescent idiopathic scoliosis. IEEE Trans Inf Technol Biomed 9: 276-282, 2005

2. Aldrich J: Correlations genuine and spurious in Pearson and Yule. Stat Sci 10: 364-376, 1995

3. Arle JE, Perrine K, Devinsky O, Doyle WK: Neural network analysis of preoperative variables and outcome in epilepsy surgery. J Neurosurg 90: 998-1004, 1999 
4. Asadi H, Kok HK, Looby S, Brennan P, O'Hare A, Thornton J: Outcomes and complications after endovascular treatment of brain arteriovenous malformations: A prognostication attempt using artificial intelligence. World Neurosurg 96: 562-569.e1, 2016

5. Azimi P, Benzel EC, Shahzadi S, Azhari S, Mohammadi HR: Use of artificial neural networks to predict surgical satisfaction in patients with lumbar spinal canal stenosis: Clinical article. J Neurosurg Spine 20: 300-305, 2014

6. Azimi P, Mohammadi HR: Predicting endoscopic third ventriculostomy success in childhood hydrocephalus: An artificial neural network analysis. J Neurosurg Pediatr 13: 426-432, 2014

7. Azimi P, Mohammadi HR, Benzel EC, Shahzadi S, Azhari S: Use of artificial neural networks to predict recurrent lumbar disk herniation. J Spinal Disord Tech 28: E161-165, 2015

8. Baumgarten $C$, Zhao $Y$, Sauleau $P$, Malrain $C$, Jannin $P$, Haegelen C: Image-guided preoperative prediction of pyramidal tract side effect in deep brain stimulation: Proof of concept and application to the pyramidal tract side effect induced by pallidal stimulation. J Med Imaging (Bellingham) 3 : 25001, 2016

9. Bonilha L, Jensen JH, Baker N, Breedlove J, Nesland T, Lin JJ, Drane DL, Saindane AM, Binder JR, Kuzniecky RI: The brain connectome as a personalized biomarker of seizure outcomes after temporal lobectomy. Neurology 84: 1846-1853, 2015

10. Celejewska A, Tukiendorf A, Miszczyk L, Składowski K, Wydmański J, Trela-Janus K: Stereotactic radiotherapy in epithelial ovarian cancer brain metastases patients. J Ovarian Res 7: 79, 2014

11. Cohen KB, Glass B, Greiner HM, Holland-Bouley K, Standridge S, Arya R, Faist R, Morita D, Mangano F, Connolly B, Glauser $\mathrm{T}$, Pestian J: Methodological issues in predicting pediatric epilepsy surgery candidates through natural language processing and machine learning. Biomed Inform Insights 8: 11-18, 2016

12. Collinger JL, Wodlinger B, Downey JE, Wang W, Tyler-Kabara EC, Weber DJ, McMorland AJC, Velliste M, Boninger ML, Schwartz AB: High-performance neuroprosthetic control by an individual with tetraplegia. Lancet 381: 557-564, 2013

13. Coumans J-V, Walcott BP, Butler WE, Nahed BV, Kahle KT: Volumetric analysis of syringomyelia following hindbrain decompression for Chiari malformation Type I: Syringomyelia resolution follows exponential kinetics. Neurosurg Focus 31: E4, 2011

14. Cuingnet R, Rosso $C$, Chupin M, Lehéricy S, Dormont D, Benali H, Samson Y, Colliot O: Spatial regularization of SVM for the detection of diffusion alterations associated with stroke outcome. Med Image Anal 15: 729-737, 2011

15. Dian JA, Colic S, Chinvarun Y, Carlen PL, Bardakjian BL: Identification of brain regions of interest for epilepsy surgery planning using support vector machines. Conf Proc IEEE Eng Med Biol Soc 2015: 6590-6593, 2015

16. Dumont TM: Prospective assessment of a symptomatic cerebral vasospasm predictive neural network model. World Neurosurg 94: 126-130, 2016

17. Dumont TM, Rughani Al, Tranmer BI: Prediction of symptomatic cerebral vasospasm after aneurysmal subarachnoid hemorrhage with an artificial neural network: Feasibility and comparison with logistic regression models. World Neurosurg 75: 57-63-28, 2011
18. Eftekhar B, Mohammad K, Ardebili HE, Ghodsi M, Ketabchi E: Comparison of artificial neural network and logistic regression models for prediction of mortality in head trauma based on initial clinical data. BMC Med Inform Decis Mak 5: 3, 2005

19. Emblem KE, Pinho MC, Zöllner FG, Due-Tonnessen P, Hald JK, Schad LR, Meling TR, Rapalino O, Bjornerud A: A generic support vector machine model for preoperative glioma survival associations. Radiology 275: 228-234, 2015

20. Erol FS, Uysal H, Ergun U, Barisci N, Serhathoglu S, Hardalac F: Prediction of minor head injured patients using logistic regression and MLP neural network. J Med Syst 29: 205-215, 2005

21. Gazit T, Andelman F, Glikmann-Johnston Y, Gonen T, Solski A, Shapira-Lichter I, Ovadia M, Kipervasser S, Neufeld MY, Fried I, Hendler T, Perry D: Probabilistic machine learning for the evaluation of presurgical language dominance. J Neurosurg 125: 481-493, 2016

22. Gornet MF, Burkus JK, Shaffrey ME, Argires PJ, Nian H, Harrell FE: Cervical disc arthroplasty with PRESTIGE LP disc versus anterior cervical discectomy and fusion: A prospective, multicenter investigational device exemption study. J Neurosurg Spine 1-16, 2015

23. Habibi Z, Ertiaei A, Nikdad MS, Mirmohseni AS, Afarideh M, Heidari V, Saberi H, Rezaei AS, Nejat F: Predicting ventriculoperitoneal shunt infection in children with hydrocephalus using artificial neural network. Childs Nerv Syst 32: 2143-2151, 2016

24. Hoffman H, Lee SI, Garst JH, Lu DS, Li CH, Nagasawa DT, Ghalehsari N, Jahanforouz N, Razaghy M, Espinal M, Ghavamrezaii A, Paak BH, Wu I, Sarrafzadeh M, Lu DC: Use of multivariate linear regression and support vector regression to predict functional outcome after surgery for cervical spondylotic myelopathy. J Clin Neurosci 22: 1444-1449, 2015

25. Hsu MH, Li YC, Chiu WT, Yen JC: Outcome prediction after moderate and severe head injury using an artificial neural network. Stud Health Technol Inform 116: 241-245, 2005

26. Hu X, Xu P, Asgari S, Vespa P, Bergsneider M: Forecasting ICP elevation based on prescient changes of intracranial pressure waveform morphology. IEEE Trans Biomed Eng 57: 1070-1078, 2010

27. Kasprowicz M, Asgari S, Bergsneider M, Czosnyka M, Hamilton R, Hu X: Pattern recognition of overnight intracranial pressure slow waves using morphological features of intracranial pressure pulse. J Neurosci Methods 190: 310318, 2010

28. Kickingereder P, Bonekamp D, Nowosielski M, Kratz A, Sill M, Burth S, Wick A, Eidel O, Schlemmer H-P, Radbruch A, Debus J, Herold-Mende C, Unterberg A, Jones D, Pfister S, Wick W, von Deimling A, Bendszus M, Capper D: Radiogenomics of glioblastoma: Machine learning-based classification of molecular characteristics by using multiparametric and multiregional MR imaging features. Radiology 281: 907-918, 2016

29. Knoll MA, Oermann EK, Yang Al, Paydar I, Steinberger J, Collins B, Collins S, Ewend M, Kondziolka D: Survival of patients with multiple intracranial metastases treated with stereotactic radiosurgery: Does the number of tumors matter? Am J Clin Oncol 2016 (Epub ahead of print)

30. Lee S, Seo S, Hwang J, Seol HJ, Nam D, Lee J, Kong D: Analysis of risk factors to predict communicating hydrocephalus following gamma knife radiosurgery for intracranial schwannoma. Cancer Med 5: 3615-3621, 2016 
31. Lo BWY, Macdonald RL, Baker A, Levine MAH: Clinical outcome prediction in aneurysmal subarachnoid hemorrhage using Bayesian neural networks with fuzzy logic inferences. Comput Math Methods Med 2013: 904860, 2013

32. Macyszyn L, Akbari H, Pisapia JM, Da X, Attiah M, Pigrish V, Bi Y, Pal S, Davuluri RV, Roccograndi L, Dahmane N, MartinezLage M, Biros G, Wolf RL, Bilello M, O'Rourke DM, Davatzikos C: Imaging patterns predict patient survival and molecular subtype in glioblastoma via machine learning techniques. Neuro-Oncol 18: 417-425, 2016

33. Malhotra K, Navathe SB, Chau DH, Hadjipanayis C, Sun $\mathrm{J}$ : Constraint based temporal event sequence mining for Glioblastoma survival prediction. J Biomed Inform 61: 267275, 2016

34. Matis GK, Chrysou OI, Silva D, Karanikas MA, Baltsavias G, Lyratzopoulos N, Baroutas S, Birbilis TA: Prediction of lumbar disc herniation patients' satisfaction with the aid of an artificial neural network. Turk Neurosurg 26: 253-259, 2016

35. McGirt MJ, Mukherjee D, Chaichana KL, Than KD, Weingart JD, Quinones-Hinojosa A: Association of surgically acquired motor and language deficits on overall survival after resection of glioblastoma multiforme. Neurosurgery 65: 463-469-470, 2009

36. Memarian N, Kim S, Dewar S, Engel J, Staba RJ: Multimodal data and machine learning for surgery outcome prediction in complicated cases of mesial temporal lobe epilepsy. Comput Biol Med 64: 67-78, 2015

37. Moher D, Liberati A, Tetzlaff J, Altman DG: Preferred reporting items for systematic reviews and meta-analyses: The PRISMA statement. BMJ 339: b2535, 2009

38. Moran A, Bar-Gad I, Bergman H, Israel Z: Real-time refinement of subthalamic nucleus targeting using Bayesian decisionmaking on the root mean square measure. Mov Disord 21: 1425-1431, 2006

39. Muniz AMS, Liu H, Lyons KE, Pahwa R, Liu W, Nobre FF, Nadal J: Comparison among probabilistic neural network, support vector machine and logistic regression for evaluating the effect of subthalamic stimulation in Parkinson disease on ground reaction force during gait. J Biomech 43: 720-726, 2010

40. Nelson DW, Nyström $H$, MacCallum RM, Thornquist B, Lilja A, Bellander B-M, Rudehill A, Wanecek M, Weitzberg E: Extended analysis of early computed tomography scans of traumatic brain injured patients and relations to outcome. J Neurotrauma 27: 51-64, 2010

41. Nelson DW, Rudehill A, MacCallum RM, Holst A, Wanecek M, Weitzberg E, Bellander B-M: Multivariate outcome prediction in traumatic brain injury with focus on laboratory values. $J$ Neurotrauma 29: 2613-2624, 2012

42. Nucci CG, De Bonis $P$, Mangiola A, Santini P, Sciandrone $M$, Risi A, Anile C: Intracranial pressure wave morphological classification: Automated analysis and clinical validation. Acta Neurochir (Wien) 158: 581-588, 2016

43. Oermann EK, Rubinsteyn A, Ding D, Mascitelli J, Starke $\mathrm{RM}$, Bederson JB, Kano $\mathrm{H}$, Lunsford LD, Sheehan JP, Hammerbacher J, Kondziolka D: Using a machine learning approach to predict outcomes after radiosurgery for cerebral arteriovenous malformations. Sci Rep 6: 21161, 2016

44. Pang BC, Kuralmani V, Joshi R, Hongli Y, Lee KK, Ang BT, Li J, Leong TY, Ng I: Hybrid outcome prediction model for severe traumatic brain injury. J Neurotrauma 24: 136-146, 2007
45. Rajpurohit V, Danish SF, Hargreaves EL, Wong S: Optimizing computational feature sets for subthalamic nucleus localization in DBS surgery with feature selection. Clin Neurophysiol 126: 975-982, 2015

46. Ranjith G, Parvathy R, Vikas V, Chandrasekharan K, Nair S: Machine learning methods for the classification of gliomas: Initial results using features extracted from MR spectroscopy. Neuroradiol J 28: 106-111, 2015

47. Rughani Al, Dumont TM, Lu Z, Bongard J, Horgan MA, Penar PL, Tranmer Bl: Use of an artificial neural network to predict head injury outcome. J Neurosurg 113: 585-590, 2010

48. Satomi J, Ghaibeh AA, Moriguchi H, Nagahiro S: Predictability of the future development of aggressive behavior of cranial dural arteriovenous fistulas based on decision tree analysis. J Neurosurg 123: 86-90, 2015

49. Scalzo F, Hamilton R, Asgari S, Kim S, Hu X: Intracranial hypertension prediction using extremely randomized decision trees. Med Eng Phys 34: 1058-1065, 2012

50. Scherer $M$, Jungk $C$, Younsi $A$, Kickingereder $P$, Müller $S$, Unterberg A: Factors triggering an additional resection and determining residual tumor volume on intraoperative MRI: Analysis from a prospective single-center registry of supratentorial gliomas. Neurosurg Focus 40: E4, 2016

51. Shi HY, Hwang SL, Lee KT, Lin CL: In-hospital mortality after traumatic brain injury surgery: A nationwide population-based comparison of mortality predictors used in artificial neural network and logistic regression models. J Neurosurg 118: 746-752, 2013

52. Valsky D, Marmor-Levin O, Deffains M, Eitan R, Blackwell KT, Bergman H, Israel Z: Stop! border ahead: Automatic detection of subthalamic exit during deep brain stimulation surgery. Mov Disord 32: 70-79, 2017

53. Wong S, Baltuch GH, Jaggi JL, Danish SF: Functional localization and visualization of the subthalamic nucleus from microelectrode recordings acquired during DBS surgery with unsupervised machine learning. J Neural Eng 6: 26006, 2009

54. Wu J, Qian Z, Tao L, Yin J, Ding S, Zhang Y, Yu Z: Resting state $\mathrm{fMRI}$ feature-based cerebral glioma grading by support vector machine. Int J Comput Assist Radiol Surg 10: 11671174, 2015

55. Yanaka K, Kamezaki T, Yamada T, Takano S, Meguro K, Nose T: Acute subdural hematoma-prediction of outcome with a linear discriminant function. Neurol Med Chir (Tokyo) 33: 552558, 1993

56. Zacharaki El, Morita N, Bhatt P, O’Rourke DM, Melhem ER, Davatzikos C: Survival analysis of patients with high-grade gliomas based on data mining of imaging variables. AJNR Am J Neuroradiol 33: 1065-1071, 2012

57. Zador Z, Sperrin M, King AT: Predictors of outcome in traumatic brain injury: New insight using receiver operating curve indices and Bayesian network analysis. PloS One 11: e0158762, 2016

58. Zheng ZS, Reggente N, Lutkenhoff E, Owen AM, Monti MM: Disentangling disorders of consciousness: Insights from diffusion tensor imaging and machine learning. Hum Brain Mapp 38: 431-443, 2017 